\title{
Quantum Theory of the Electron Transfer Coefficient
}

\author{
Manuel González-Pérez
}

\begin{abstract}
Department of Postgraduate in Biomedical Engineering Sciences of the Universidad Popular Autónoma del Estado de Puebla (UPAEP).

17 sur 901. Colonia Santiago. Puebla, México
\end{abstract}

\begin{abstract}
In the recent years, quantum chemistry has taken an essential place in the prediction of molecular interactions, in both for chemical reactions and for solutions. The primary objective of this work is to present a new theory of the electron transfer coefficient (ETC) one molecule to another.As a result of the application of this theory, examples are presented in which theoretical calculations coincide with the results of laboratories of already published works.
\end{abstract}

Keywords-Quantum chemistry, Coefficient of electron transfer, HOMO, LUMO, Electrostatic potential.

\section{INTRODUCTION}

Van der Waals forces (VWF) non-covalent or dispersing forces are said to be ubiquitous and influence the structure, stability, dynamics, and function of molecules and materials through chemistry, biology, physics and the science of materials. It is also stated that these forces are of mechanical quantum origin and arise from electrostatic interactions between fluctuations in electronic charge density. Experienced scientists thoroughly explore mathematical concepts and procedures necessary for an accurate treatment of VWF interactions, and a systematic and unified framework for classifying the first current principles for calculating VWF. ${ }^{[1]}$

Some authors define the banned band or bandgap (BG) as the separation between HOMO-LUMO between molecules of the same chemical species. They tell us that in general for polycyclic aromatic hydrocarbons a BG $<1.3$ has a very high chemical reactivity. ${ }^{[2]}$

\subsection{Use of wave and density functions and their} underlying concepts.

Recent works show the use of "time-dependent functional theory" (TDFT) ofthe "theory of the density" (TD) and proper BG identification, to reproduce known experimental data, and to predict new unknown data.Due to these realized works, arises the necessity to connect the atomic-molecular quantum theory and the theory of the functional density. ${ }^{[3]}$ Also, investigators can rationalize and suggest ways of practically reconciling observed discrepancies. ${ }^{[4]}$
A combined experimental and computational study of a series of substituted pentacenes including halogenated, phenylated, silylethynylated and thiolated derivatives is presented. Experimental studies include the synthesis and characterization of six new and six known pentacene derivatives and a kinetic study of each derivative under identical photooxidative conditions. The combined results provide for the first time a quantitative evaluation of HOMO-LUMO voids and photooxidative resistances for a large number of pentacene derivatives as a function of the substituents. Silylethynyl-substituted pentacenes have small HOMO-LUMO gaps, but they are not the longestlived species under photooxidative conditions, contrary to widespread perception. ${ }^{[5]}$

Other researchers analyzed the significant intermolecular interactions between a drug used in the treatment of 5fluorouracil cancer and human epidermal growth factor (a major cancer-related biological complex). Correctly, a careful evaluation of the significant structural, spectroscopic and electronic properties involved in the interaction was carried out. For the maximum $\lambda$ of the theoretical calculation $(254.89 \mathrm{~nm})$ of $\mathrm{UV} / \mathrm{Vis}$, the electronic transition from HOMO to LUMO occurs at $4.89 \mathrm{eV}^{[6]}$

In other studies, HOMO-LUMO void extrapolation for $\pi$ conjugated oligomers at the theory level accurately predicts (within 0.1-0.2 eV) the conjugated polymer BG only when they are long (at least 20 monomers) $\pi \cdot{ }^{[7]}$ Conjugated oligomers are used for extrapolation. This twisted conformation allows the intercalation of the relatively high $\pi(\mathrm{B}=\mathrm{B})$ orbital and the low $\pi *$ orbital of the anthill rest without significant conjugation, resulting in a small HOMO-LUMO space and finally a $\mathrm{CH}$ borylation of the anthryl unit. ${ }^{[8]}$

On the other hand, two approaches are studied: a) wave function theory (WFT), and b) density functional theory (DFT) are the two most popular solutions to the problems of theelectronic structure of atoms and molecules. The results provide convincing evidence suggesting that WFT and DFT are complementary to each other, to understand the properties of electronic structure and molecular reactivity from different perspectives. Therefore, there 
must be a bridge or bridges between the two approaches. ${ }^{[9]}$

The ultraviolet absorption spectrum of 2,6-dichloro-3nitropyridine $(2,6,3 \mathrm{DCNP})$, has been carried out experimentally (in water, $\mathrm{n}$-hexane, ethanol, methanol and $\mathrm{MgO}$ ) and theoretically (in water, ethanol, and methanol) in the range $3500-2300 \mathrm{~cm}^{-1}$ in the solution phase. The electro-absorption spectra predicted from the computation of the functional theory of density of the functional theory of time-dependent density (TD-DFT) have been analyzed and compared with the experimental UV-Vis spectrum. Excellent results were obtained from this comparison. ${ }^{[10]}$ In this way, it is demonstrated that quantum chemical techniques, such as DFT have become a powerful tool in the investigation of molecular structure and vibrational spectrum and are finding increasing use in applications related to biological systems. Also, the experimental results are in line with theoretical calculations and provide positive evidence through themolecular coupling. In most cases, vibration assignments and calculation of Potential Energy Distribution (PED) are carried out using software of different types and calculating the molecular electrostatic potential (MEP) for a given compound can predict reactive sites for the electrophilic and nucleophilic attack. Correctly, the calculated HOMO and LUMO energies not only show the transfer of charge within the molecule but also in interaction with another molecule of the same chemical species or another different chemical species. $^{[11,12]}$

In calculations of the stability of the molecule derived from the hyperconjugative interactions, the offlocalization of charge has been analyzed using natural bond orbital analysis (NBO). In the case of a first order hyperpolarization, they are calculated with the MEP and Fukui functions. ${ }^{[13]}$

In a real molecule, charge migration competes with charge transfer, so nuclear movement also results in redistribution of charge.Study this competence; quantum dynamics simulations are used. ${ }^{[14]}$

Three rules for creating highly efficient unimolecular rectifiers using asymmetric anchor groups have been proposed by Van Dyck and Ratner. ${ }^{[15]}$

The objective of this work is to present a new theory of the coefficient of electron transfer that serves to calculate something like "impedance" at the molecular level. It must be a bridge between the value of the band and the value of the electrostatic potential.

\section{METHODOLOGY: THE CREATION OF A NEW THEORY}

2.1 First moment of molecular interaction.

2.1.1Electrostatic Potential (EP):
Postulate 2.1.1.1there is a positive pole and a negative pole in each molecule.

Postulate2.1.1.2there is an electrostatic potential calculable by quantum methods at each pole.

Hypothesis2.1.1.1 the electrostatic potential (EP) relative to the interaction of two molecules is the absolute value of the difference of the electrostatic potentials of each pole.

2.2Second moment of molecular interaction.

2.2.1Molecular Orbital Theory (MOT):

Postulate2.2.1.1there is an Orbital filled with electrons (HOMO).

Postulate 2.2.1.2there is a calculable electrondeficient Orbital (LUMO).

Hypothesis 2.2.1.1The Bandgap (BG) is the absolute value of the difference of the calculated values of HOMO and LUMO.

Hypothesis 2.2.1.2The BG is the value of the energy that an electron (electronic cloud) needs to jump from one molecule to another.

2.3 Electron Transfer Coefficient (ETC):

Hypothesis 2.3.1the ETC is the ratio of dividing the relative value of the $B G$ between the relative values of the EP.

Hypothesis 2.3.2 the ETC value indicates the number of times the electron needs its EP to jump from HOMO of a molecule $A_{1}$ to LUMO of a molecule $A_{2}$.

Hypothesis 2.3.3 Cross Band. The ETC value indicates the number of times the electron needs its EP to jump from HOMO of a molecule $A_{1}$ of the chemical species 1 to LUMO of a molecule $B_{2}$ of the chemical species 2.

Corollary 2.3.1the electron jumps down the path that has the least energy or lower ETC value.

\section{RESULTS AND DISCUSSION}

3.1 The first results.

The result of the hypothesis 2.1.1.1 is the equation 3.1. It is necessary to subtract the value of the positive pole with the negative pole and to take the absolute value.

$E P=\left|E_{-}-E_{+}\right|$

As a result of the hypothesis 2.2.1.1,itobtains equation 3.2.

$B G=|H O M O-L U M O|$

The hypothesis 2.3.1 generates the equation 3.3.

$E T C=\frac{B G}{E P}$

3.2 Seconds results.

In the first place, I must recognize the enormous quantum scientists who formulated the whole theory of chemistry 
and physics. Moreover, on the other hand also my recognition to the great geniuses of the computer. With this latest science, we are moving forward with strides in quantum science and methodology.

3.2.1 Pure substances.

The first thing that was studied was the interaction between two molecules of a pure substance. Later, more and more molecular interactions were studied.

The interpretation of ETC values of the interaction of two molecules of the same substance related to reactivity and chemical stability.

Hypothesis 3.2.1.1 the chemical stability of the interaction between two molecules of the same pure substance is represented by the lower ETC value of both.

Corollary 3.2.1.1.1 As a consequence of the previous hypothesis (3.2.1.1), the higher value of ETC represents a higher reactivity of the pure substance when it interacts with a molecule of another substance.

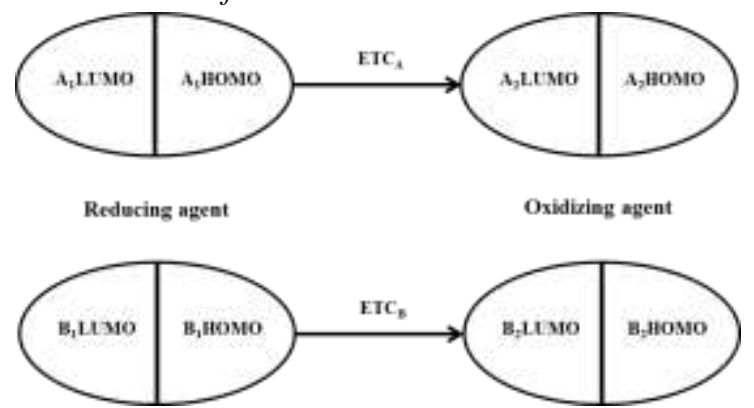

Fig. 3.1: Interaction between two molecules of the samepure substance $\left(A_{1}: A_{2}\right)$. The higher value of ETC represents a more significant reactivity of the pure substance with a third molecule of another pure substance that is present $(B)$.

3.2.2Cross bands of pure substances

Hypothesis 3.2.2.1 A cross band is carried out when the HOMO of a substance $A_{l}$ prefers the LUMO of a substance $B_{1}$ or vice versa. The primary cause is because nature seeks the lowest energy path.

Corollary 3.2.2.1.1 As a consequence of the previous hypothesis. The electron (electron cloud) prefers the path of least energy or less resistance.

Corollary 3.2.2.1.2 As a consequence of the previous corollary, ETC represents an opposition to the displacement of the electron. This opposition is equivalent to the impedance in the electronic theory.

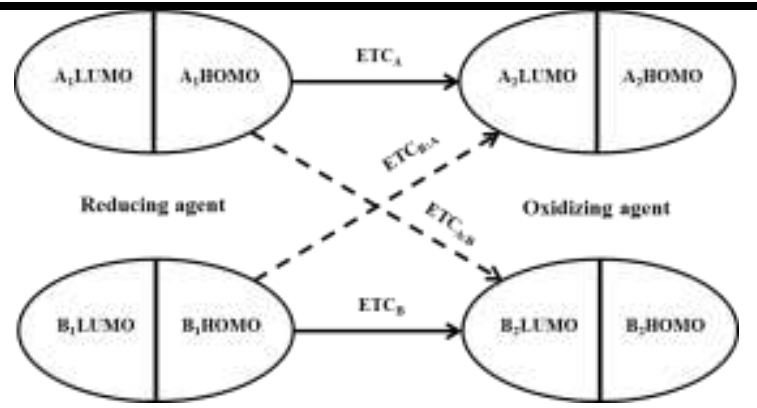

Fig. 3.2 Cross band. A cross band is carried out when the HOMO of a substance $A_{1}$ prefers the LUMO of a substance $B_{1}$ or vice versa. The leading cause is because nature seeks the lowest energy path.

\subsubsection{Quantum wells}

In Figure 1, the dotted lines represent the bottoms of the quantum wells of the pure substances. The points represent the two possible combinations: $\mathrm{A}_{\mathrm{HOMO}} \mathrm{vs.}$ $\mathrm{B}_{\text {LUMOand vice versa. }}$

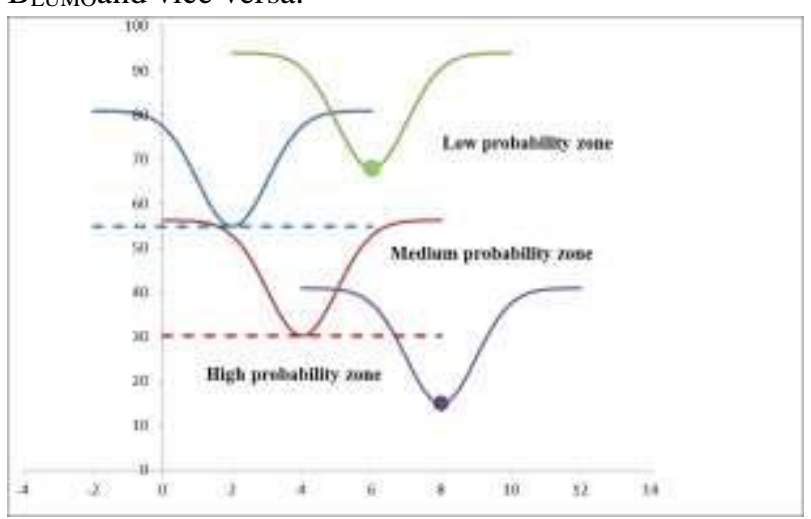

Fig. 1: Quantum wells

The dotted lines represent the bottom of each well of the pure interacting substances. Single points represent the bottom of each cross-band well.

Hypothesis 3.2.3.1there are two possibilities when crossing the bands of the molecules from $A_{\text {Номо }}$ to $B_{L U M O}$ and vice versa $B_{\text {Hомо }}$ to $A_{L U M O}$.

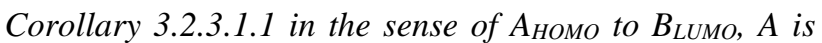
said to be a reducing agent, while $B$ is an oxidizing agent.

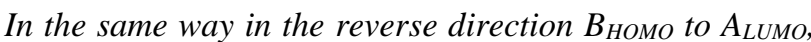
it is said that $B$ is a reducing agent, whereas $A$ is an oxidizing agent.

Corollary 3.2.3.1.2the mechanism for electrostatic potential (EP) is identical.

Hypothesis 3.2.3.2 there are two limits to placing the bottoms of the quantum wells in a two-dimensional diagram. These two limits correspond to the ETCs of each of the substances that are mixed.

Corollary 3.2.3.2.1 As a consequence of the previous hypothesis, three zones of probabilities of molecular interaction is generated: 
a) Zone of high probability. This zone is below the lower limit.

b) Zone of medium probability. This zone is located in the lower limit and the upper limit.

c) Zone of low probability. This zone is located above the upper limit (fig. 3.3).

Corollary 3.2.3.2.2the $x$-axis has arbitrary units in the two-dimensional quantum well diagram. The y-axis represents the value of ETCs.

3.2.3Examples

3.2.3.1 Aflatoxin B1 as a carcinogen.

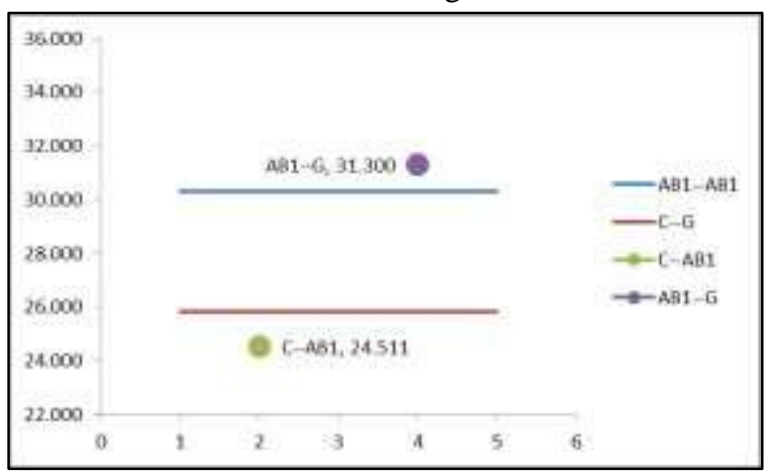

Fig. 2: Aflatoxin B1

In this example, it is observed that the CytosineGuanine $(C-G)$ pair of the DNA is strongly oxidized by Aflatoxin $B 1$ (AB1). The quantum wells were not drawn to simplify the figure.

The ETC value of the interaction of Aflatoxin B1 and the C-G pair of DNA indicates in this figure of quantum wells is located in high probability zone.

In contrast, the ETC value of the reverse direction, i.e., aflatoxin B 1 behaves as an antioxidant for the $\mathrm{C}-\mathrm{G}$ pair is avery low probability. ${ }^{[16]}$

3.2.3.2 Methylenedioxymethamphetamine and Adrenalin Figure 3 shows the interaction ofmethylenedioxymethamphetamine (MDMA) vs. adrenalin. The smaller ETC is given when the HOMO electrons of the MDMA jump to the LUMO of adrenaline.The probability of interaction from these two molecules is very high.

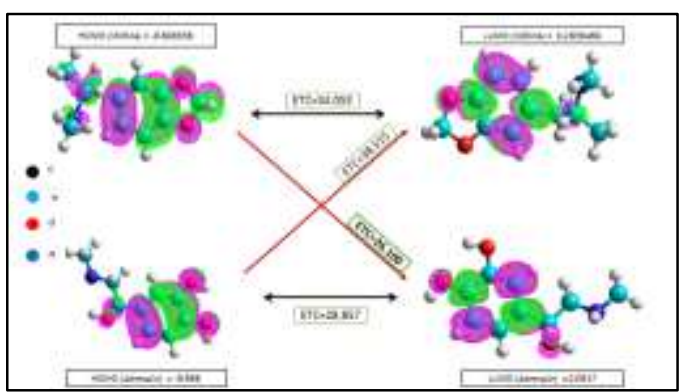

Fig. 3: Methylenedioxymethamphetaminevs. Adrenalin.

Cross Band. The probability of interaction from these two molecules is very high
3.2.3.3 Other examples.

Nicotine, Neurotransmitter, Glucose, and others. ${ }^{[18]}$

\section{CONCLUSION}

I present a new theory for calculating the electron transfer coefficient between molecules.

The essence of the theory is to calculate how many times an electron or electronic cloud needs its electrostatic potential to jump from the HOMO of one molecule to the LUMO of another molecule. This calculated value is called the electron transfer coefficient (dimensionless). This concept of ETC is similar to electronic impedance. There is evidence that this theory agrees with laboratory experiments, both in the current literature and in the historical literature.

\section{ACKNOWLEDGEMENTS}

Recognition with adeep respect for all those giants of quantum physics and quantum chemistry. With the same depth of respect go this recognition for the great geniuses of the sciences and computational technologies.

\section{REFERENCES}

[1] Hermann, J., \& Tkatchenko, A. (2017). Firstprinciples models for van der Waals interactions in molecules and materials: concepts, theory, and applications. Chemical Reviews, 117, 4714-4758.

[2] Aihara, J. I. (1999). Reduced HOMO- LUMO gap as an index of kinetic stability for polycyclic aromatic hydrocarbons. The Journal of Physical Chemistry A, 103(37), 7487-7495.

[3] Matta, C. F. (2017). On the connections between the quantum theory of atoms in molecules (QTAIM) and density functional theory (DFT): A letter from Richard FW Bader to Lou Massa. Structural Chemistry, 1-7.

[4] Zdetsis, A. D., \& Economou, E. N. (2017). Rationalizing and reconciling energy gaps and quantum confinement in narrow atomically precise armchair graphene nanoribbons. Carbon, 116, 422434.

[5] Kaur, I., Jia, W., Kopreski, R. P., Selvarasah, S., Dokmeci, M. R., Pramanik, C., ... \& Miller, G. P. (2008). Substituent effects in pentacenes: Gaining control over HOMO- LUMO gaps and photooxidative resistances. Journal of the American Chemical Society, 130(48), 16274-16286.

[6] Almeida, M. O., Barros, D. A., Araujo, S. C., Faria, S. H., Maltarollo, V. G., \& Honorio, K. M. (2017). Study on molecular structure, spectroscopic properties (FTIR and UV-Vis), NBO, QTAIM, HOMO-LUMO energies and docking studies of 5fluorouracil, a substance used to treat cancer. 
Spectrochimica Acta Part A: Molecular and Biomolecular Spectroscopy, 184, 169-176.

[7] Zade, S. S., \& Bendikov, M. (2006). From oligomers to polymer: convergence in the HOMO- LUMO gaps of conjugated oligomers. Organic letters, 8(23), 5243-5246.

[8] Wang, S., Arrowsmith, M., Böhnke, J., Braunschweig, H., Dellermann, T., Dewhurst, R., ... \& Thiess, T. (2017). Engineering a Small HOMO-LUMO Gap and Intramolecular C-H Borylation by Diborane/Anthracene Orbital Intercalation. Angewandte Chemie International Edition.

[9] Huang, Y., Rong, C., Zhang, R., \& Liu, S. (2017). Evaluating frontier orbital energy and HOMO/LUMO gap with descriptors from density functional reactivity theory. Journal of molecular modeling, 23(1), 3.

[10] Kumar, S., Yadav, M. K., \& Teotia, J. (2017). Solvent Effect on Electronic Transitions, Homo Lumo Analysis of 2, 6-Dichloro-3-Nitro Pyridine Under Hartree-Fock and Density Functional Theory. In Recent Trends in Materials and Devices (pp. 509517). Springer International Publishing.

[11] Kuruvilla, T. K., Prasana, J. C., Muthu, S., George, J., \& Mathew, S. A. (2017). Quantum mechanical and spectroscopic (FT-IR, FT-Raman) study, NBO analysis, HOMO-LUMO, first-order hyperpolarizability and molecular docking study of methyl [(3R)-3-(2-methylphenoxy)-3-phenylpropyl] amine by density functional method. Spectrochimica Acta Part A: Molecular and Biomolecular Spectroscopy, 188, 382-393.

[12] Muthu, S., Prasanna, J. C., \& Bharathy, G. (2017). Vibrational Spectra, NBO, HOMO-LUMO and Molecular Docking Studies of Diclofenac based on Density Functional Theory. Asian Journal of Research in Social Sciences and Humanities, 7(5), 63-74.

[13] Raja, M., Muhamed, R. R., Muthu, S., \& Suresh, M. (2017). Synthesis, spectroscopic (FT-IR, FT-Raman, NMR, UV-Visible), NLO, NBO, HOMO-LUMO, Fukui function and molecular docking study of (E)-1(5-bromo-2-hydroxybenzylidene) semicarbazide. Journal of Molecular Structure, 1141, 284-298.

[14] Spinlove, K. E., Vacher, M., Bearpark, M., Robb, M. A., \& Worth, G. A. (2017). Using quantum dynamics simulations to follow the competition between charge migration and charge transfer in polyatomic molecules. Chemical Physics, 482, 52-63.

[15] Thong, A., Shaffer, M. S. P., \& Horsfield, A. (2017). HOMO-LUMO coupling: the fourth rule for highly effective molecular rectifiers. Nanoscale.
[16] Gonzalez-Perez, M. (2017) Quantum modeling to determine the carcinogenic potential of aflatoxinB1 produced by Aspergillus sp and its metabolic derivate aflatoxin M1.Mexican Journal of Biotechnology 2017, 2(2):255-270.

[17] Mondragón-Jiménez, J. F., Ojeda-Lara, B., AlvarezAguilar, A., Brito-Pérez, C. A., Rosales-Hernández, F. J., \& González-Pérez, M. (2017). INTERACTION OF METILENDIOXIMETANFETAMINA VS. NEUROTRANSMITTERS AND THE RELATIONSHIP BY THE QUANTUM METHOD.WORLD JOURNAL OF PHARMACY AND PHARMACEUTICAL SCIENCES. Volume 6, Issue 8, 2467-2477.

[18] https://www.researchgate.net/profile/Manuel_Gonzal ez-Perez/contributions. 